ISSN: 1021-7444

\title{
EVALUACIÓN ECONÓMICO-PRODUCTIVA DE UN SISTEMA DE PRODUCCIÓN DE LECHE EN EL TRÓPICO ${ }^{1}$
}

\author{
Juan Prisciliano Zárate-Martínez², Valentín Alberto Esqueda-Esquivel ${ }^{3}$, Julio Cesar Vinay-Vadillo², \\ Sergio Miguel Jácome-Maldonado
}

\begin{abstract}
RESUMEN
Evaluación económico-productiva de un sistema de producción de leche en el trópico. El trabajo se realizó en el Campo Experimental La Posta, en Paso del Toro, municipio de Medellín de Bravo, Veracruz, México. El objetivo fue determinar el comportamiento productivo y económico de un sistema tropical especializado (SLT). Este es un sistema de producción de leche en el trópico con ganado Holstein (Hs), Suizo Pardo (SP) y sus cruzas con cebú (C). El genotipo, sexo, época, peso al nacimiento (PNAC), ganancia diaria de peso (GDP) al destete GDP1, GDP2 destete-año, GDP3 año18 meses, edad a la concepción (ECONC), días en lactancia, producción/lactancia (PL) y su error estándar de la media (EEM), se utilizaron para evaluar el comportamiento productivo. La inversión inicial, egresos, ingresos, costos variables (CV), utilidad neta, rentabilidad (R), costos totales, punto de equilibrio y margen de ganancia (MG) para el financiero. El mayor $(\mathrm{P}<0,05)$ peso al nacimiento de machos fue el genotipo 80-99\% SP (41,62 kg; EEM 2,85); la menor ( $<<0,05)$ GDP1 de hembras fue las SP $(0,423 \mathrm{~kg}$; EEM 0,083). La ECONC fue $(\mathrm{P}<0,05)$ a favor de los genotipos SP y $50 \%$ Hs $(25,73$; EEM 4,20 y 26,67; EEM 9,082 meses, respectivamente). La mejor PL $(\mathrm{P}<0,05)$ para el genotipo 80-99\% Hs (3796 litros; EEM 246,35). La inversión inicial fue de US\$78660.5, los conceptos que más impactaron para CV fueron; alimentación (26,24\%) y mano de obra (16,70\%). La utilidad neta fue de US\$2971.6 y su relación beneficio costo de 1:1.11. Un SLT puede alcanzar 4000 litros por lactancia y ser autofinanciable.
\end{abstract}

Palabras claves: Leche, trópico, grupos genéticos.

\begin{abstract}
Economic and productive evaluation of a tropical dairy unit. This study was conducted at Campo Experimental La Posta, in Paso del Toro, Medellín de Bravo, Veracruz, México. The objective was to assess the economic and productive behavior of a specialized tropical dairy system (TDS). The dairy system included Holstein (Hs), Brown Swiss (BS) and Cebu crosses (C) cattle. Genotype, sex, season, weight at birth (BW), daily weight gain (DWG) at weaning GDP1, GDP2 weaning-year, GDP3 year-18 months, age at conception, days in lactation, production/ lactation, and the mean standard error for each (EEM), were utilized to determine the productive performance. Initial investment, income, expenses, variable costs (CV), net gains, profitability $(\mathrm{P})$, total production cost, equilibrium point, and profit margin $(\mathrm{PM})$ were considered for financial analysis. The highest BW $(\mathrm{P}<0.05)$ in males was for the genotype 8099\% BS (41.62 kg; MSE 2.95); the lowest GDP1 ( $\mathrm{P}<0.05)$ in females was of BS (423 kg; MSE 0.08). ACONC showed differences $(\mathrm{P}<0.05)$ that favored genotypes BS and $50 \% \mathrm{Hs}$ (25.73 and 26.67 months; MSE 4,205 and 9.08 respectively). The best PL $(\mathrm{P}<0,05)$ was for the genotype $80-99 \%$ Hs $(3796$ L; MSE 246.35). Initial investment was US\$78660.5; the factors that most impacted CV were feed (26.24\%) and labor (16.70\%). Net gain was of US\$2971.6, and Its cost:benefit ratio was $1: 1.11$. A TDS can achieve a production of $4000 \mathrm{~L}$ per lactation and be self-financing.
\end{abstract}

Key words: Milk, tropic, genetic groups.

\footnotetext{
1 Recibido: 3 de marzo, 2010. Aceptado: 22 de noviembre, 2010. Proyecto productivo Sistema de Producción de Leche en el Trópico. CIRGOC. Instituto Nacional de Investigaciones Agrícolas y Pecuarias (INIFAP).

2 Campo Experimental La Posta. Instituto Nacional de Investigaciones Agrícolas y Pecuarias (INIFAP). Km 22.5 carretera Veracruz-Córdoba, Col. Paso del Toro, municipio de Medellín de Bravo, Ver., México. zarate.juan@inifap.gob.mx; vinay.julio@inifap.gob.mx

3 Campo Experimental Cotaxtla. Instituto Nacional de Investigaciones Agrícolas y Pecuarias (INIFAP). Ocampo No. 234 Despacho 322 , Veracruz, Ver. Correo electrónico: esqueda.valentin@inifap.gob.mx; jacome.sergio@inifap.gob.mx.
} 


\section{INTRODUCCIÓN}

En el continente Americano se producen alrededor de 140 millones de toneladas de leche $(25 \%$ de la producción mundial), pero América Latina sólo contribuye con 53 millones de toneladas. Uruguay, Argentina y Costa Rica son los únicos países autosuficientes de la región (Loyola 2000), mientras que algunos, principalmente México, Brasil, Perú, Venezuela y Cuba, importan 800000 toneladas de leche en polvo. En Estados Unidos y Canadá se mantiene una disponibilidad de 120 litros de leche/persona/año, pero en los países de Centro América y el Caribe, ésta se reduce a 58 litros (Ponce 2000).

La producción de leche en México sólo cubre el $65 \%$ de la demanda interna; a principios y mediados de los 90's, la producción promedio fue de 7474 millones de litros por año (García et al. 1997), la cual se incrementó a 8354 millones de litros a finales de la década (Ponce 2000) y a 10183 millones de litros en el año 2007, en el que el valor de la producción ascendió a 39919 millones de pesos (SAGARPA 2004). El consumo actual de leche en México es de 97 litros por persona, cantidad que equivale a la mitad de lo que recomienda la Organización de las Naciones Unidas para la Agricultura y la Alimentación.

En México se reconocen cuatro sistemas de producción de leche: 1 . Sistema especializado, que es el más importante, y contribuye con $50,6 \%$ de la producción nacional, 2. Sistema semi-especializado, con una participación de $21,3 \%$, 3. Sistema doble propósito, con $18,3 \%$ y 4 . Sistema familiar, con $9,8 \%$ (SAGARPA 2004).

La región tropical ofrece las mejores perspectivas para incrementar la producción de leche en el país (Rojas 1996); sin embargo, en las explotaciones lecheras se utiliza de forma ineficiente el activo fijo (tierra y ganado) y existe un rechazo al uso de tecnología que implique mayores gastos (Castro et al. 2001). Dadas las condiciones actuales en las que se encuentra la ganadería de México, es necesario hacer de esta actividad un negocio rentable.

Existen pocos estudios de tipo económico-productivo que permitan estimar la situación y características que presentan las explotaciones lecheras en las zonas tropicales del país. Para obtener esa información, se requiere el registro sistemático de los gastos, los ingresos o venta de los productos y los parámetros productivos y reproductivos. Lo anterior proporciona una visión clara sobre la situación de este sistema de producción, y permite verificar los factores de la producción que afectan su rentabilidad (Rojas 1996, Nuñez et al. 1999).

En la actualidad, la reducción de los costos de producción es un objetivo de supervivencia para toda la lechería de la región, y por ello, se requiere la búsqueda de sistemas más eficientes. Este trabajo se realizó con el objetivo de determinar el comportamiento productivo y económico de un módulo de lechería tropical especializada durante un periodo de dos años.

\section{MATERIALES Y MÉTODOS}

Se utilizaron los registros de animales cruzados Holstein por Cebú y Suizo Pardo por Cebú con un procedimiento de encaste (120 lactancias completas) y datos económicos generados durante los años 2002 y 2003 en el Módulo de Lechería Tropical Especializada del Campo Experimental La Posta del Instituto Nacional de Investigaciones Forestales, Agrícolas y Pecuarias (INIFAP), localizado en el km 22,5 de la carretera Veracruz-Córdoba, en Paso del Toro, municipio de Medellín de Bravo, Veracruz, México, a 15 18' latitud Norte, $96^{\circ} 10^{\prime}$ longitud oeste y una altitud de $12 \mathrm{~m}$ sobre el nivel del mar. La región tiene un clima tropical subhúmedo $A w_{1}$, con temperatura máxima, media y mínima de $35,3,25$ y $15^{\circ} \mathrm{C}$, respectivamente, una precipitación pluvial media anual de $1461 \mathrm{~mm}$ y humedad relativa de 77,4\% (García 1989).

La infraestructura consistió en cuatro corrales para vacas en producción, un corral para vaquillas y vacas secas, cinco corraletas para vacas próximas al parto, una sala con 12 becerreras, un corral para becerras en desarrollo, una bodega de insumos, un corral de manejo, una sala de ordeña de seis plazas, un tanque frío y lechería.

Las praderas estaban establecidas con pasto Estrella de África (Cynodon plectostachyus) y se manejaron bajo un sistema de pastoreo rotacional nocturno en 27 divisiones de un cuarto de hectárea con cercos eléctricos, donde se alternaron periodos de ocupación cortos con descanso de 30 a 45 días en época lluviosa y seca, respectivamente. Para fertilizar los potreros se aplicaron $400 \mathrm{~kg}$ de nitrógeno $\left(\mathrm{N}_{2}\right)$ por hectárea, fraccionado en seis aplicaciones mensuales de $50 \mathrm{~kg}$ 
(junio-noviembre) y $100 \mathrm{~kg}$ de fósforo $\left(\mathrm{P}_{2} \mathrm{O}_{5}\right)$, en una sola aplicación al inicio de las lluvias. Como fuente de $\mathrm{N}_{2}$ se utilizó urea y de P, superfosfato de calcio triple. Además, durante la época de sequía las praderas se regaron por gravedad. El gusano falso medidor (Trichoplusia ni) se controló con la aplicación de metamidofós durante el mes de septiembre y la mosca pinta o salivazo (Aeneolamia postica), con pastoreo intensivo. El control de malezas se realizó durante todo el año en forma manual o con aplicación de la mezcla formulada de picloram + 2,4-D durante los meses de febrero a junio. Se utilizaron ocho hectáreas para producción de forraje de sorgo forrajero (Sorghum bicolor cv Beefbuilder) para ensilar. Para la preparación del lote se realizó un barbecho y un rastreo un mes antes del inicio de lluvias y se dio otro rastreo al iniciarse las lluvias; posteriormente se sembró el sorgo y 15 días después de la siembra, se fertilizó con $138 \mathrm{~kg}$ de $\mathrm{N}_{2} \mathrm{y}$ $69 \mathrm{~kg} \mathrm{P}_{2} \mathrm{O}_{5}$ por hectárea y $69 \mathrm{~kg}$ de $\mathrm{N}_{2}$ por hectárea, cuando la planta presentó una altura entre 40 y $50 \mathrm{~cm}$; el corte y ensilado se realizó cuando el grano estaba en estado lechoso-masoso.

En general se estimó que con el manejo de la pradera descrito y la producción de forraje para ensilar se obtendrá una capacidad de carga de cuatro unidades animales (UA)/ha, con una capacidad total de 102 UA en el sistema.

Se aplicó el mismo manejo y programa de alimentación a los diferentes grupos genéticos, los cuales se trataron por etapa productiva o fisiológica, lo que generó los siguientes grupos: becerros (as) del nacimiento al destete (90 días); becerras del destete a los 180 días de edad; novillonas de 181 días a 18 meses de edad (340 kg); vacas secas y vaquillas de inicio de manejo reproductivo al parto y vacas en producción.

\section{Becerros (as) del nacimiento al destete (90 días)}

Se controló que los becerros mamaran calostro durante las tres primeras horas de vida y se les desinfectó el cordón umbilical con una solución de yodo al 3\%. Durante los cinco primeros días posteriores al nacimiento permanecieron con sus madres, y después se alojaron en corraletas individuales en la sala de crianza (Barradas et al. 1979), donde se identificaron mediante tatuaje en el pabellón interno de las orejas, se descornaron con pastas a base de sosa caústica, se les aplicó vitamina ADE por vía intramuscular y se extirparon las tetas extras a las hembras. Posteriormente se elaboró una tarjeta de registro individual para asentar todos los eventos y datos productivos. La crianza fue artificial desde el sexto día hasta el destete y la alimentación consistió en cuatro litros de leche entera, divididos en dos tomas (mañana y tarde), más alimento concentrado con $18 \%$ de proteína cruda (PC) y $70 \%$ de total de nutrientes digestibles (TND) a voluntad; también se proporcionó heno de pasto Pangola (Digitaria decumbens) a voluntad para estimular el desarrollo del rumen (Román et al. 1970). El destete se efectuó a los 90 días de edad y se registró el peso de todos los animales mensualmente. Los machos salieron del sistema de lechería y se llevaron a potreros permaneciendo en pastoreo y vendidos al alcanzar un peso vivo de alrededor de los $290 \mathrm{~kg}$, con un precio promedio de $\$ 14,80 / \mathrm{kg}$.

\section{Becerras del destete a los 180 días de edad}

Después del destete las becerras se llevaron a un potrero de 1,4 ha. Durante las horas de mayor radiación solar se alojaron en un establo provisto con comederos y bebederos, donde se les proporcionaron $2 \mathrm{~kg}$ diarios del alimento concentrado, agua, sales minerales y ensilaje de sorgo a voluntad; inmediatamente después del destete se les aplicó vitamina ADE por vía intramuscular (Ochoa 1979). Se realizaron desparasitaciones internas o externas (de acuerdo al producto utilizado) cuatro veces al año, previo pesaje; se vacunó contra clostridiasis en los meses de marzo y septiembre, y contra derriengue (virus Lyssavirus) en agosto; no se vacunaron las hembras contra brucelosis por tener un hato libre de esta enfermedad. Se registró el peso de todos los animales mensualmente.

\section{Novillas de 181 días a 18 meses de edad $(340 \mathrm{~kg})$}

Se manejaron en un potrero de tres hectáreas. Se les suplementó con sales minerales y agua a libertad, y se les aplicó vitamina ADE por vía intramuscular al inicio de la sequía. Se realizaron desparasitaciones internas y externas (de acuerdo al producto utilizado) cada tres meses, previo pesaje; se vacunó contra clostridiasis en los meses de marzo y septiembre y contra derriengue en agosto. Después de esta etapa se 
inicia su manejo reproductivo, y pasan a formar parte del siguiente grupo (Reyes et al. 1999). Se registró el peso y los eventos reproductivos de todos los animales mensualmente.

\section{Vacas secas y vaquillas de inicio de manejo repro- ductivo al parto}

Se manejaron como seguidoras en pastoreo rotacional nocturno en un sistema de líderes y seguidoras; se suplementaron con $2 \mathrm{~kg}$ de concentrado con $16 \%$ de PC y $70 \%$ de total de nutrientes digestibles (TND) ani$\mathrm{mal} /$ día, la cantidad de alimento concentrado se ajustó para que las vacas alcanzaran una condición corporal de 3,5 al parto (Edmonson et al. 1989). Se ofrecieron sales minerales y agua a libertad; además, se les proporcionó ensilaje de sorgo durante el día. En las vacas secas se realizó una desparasitación interna antes del parto, se realizaron baños garrapaticidas y la vacunación contra derriengue se hizo en el mes de agosto. Para las vaquillas, se realizó desparasitación interna cada seis meses, vacunación contra clostridiasis en los meses de marzo y septiembre, y contra derriengue en el mes de agosto. Durante el manejo reproductivo de las vacas al secado, se diagnosticó la gestación por palpación rectal; las vaquillas también se revisaron por vía rectal para constatar el desarrollo y funcionamiento de sus órganos genitales, y se detectaron los estros dos veces al día (por la mañana y tarde).

\section{Vacas en producción}

Las vacas en producción se manejaron en pastoreo rotacional nocturno en el sistema de líderes y seguidoras; para facilitar el manejo durante el ordeño y el encierro, se mantuvieron en dos grupos: vacas recién paridas al quinto mes de lactancia y vacas del quinto mes de lactancia al secado. El ordeño (22 vacas promedio en ordeño semana/año) se hizo en forma mecánica dos veces al día (de 6 a 8 am y de 4 a 6 pm), y la producción del ordeño se midió y registró en hojas semanales. La suplementación consistió en 1,5 kg de alimento balanceado con $16 \%$ de PC y $70 \%$ de TND por ordeña/animal/día, y 350 g más por kilogramo, a partir del sexto kilo de leche producido por vaca (Rodríguez et al. 1999), el cual se ofreció en forma individual, encadenando las vacas al comedero del establo; también se proporcionaron sales minerales y agua a libre consumo, más $20 \mathrm{~kg}$ de ensilaje de sorgo por animal durante el encierro. El control sanitario consistió en vacunación contra derriengue en el mes de agosto, baños garrapaticidas periódicos y pasaje por un pediluvio con una solución de sulfato de cobre al 5\%, durante la época de lluvias. Las vacas se ordeñaron en el orden siguiente: primero las vacas de una semana de paridas al quinto mes de lactancia, después las vacas del quinto mes de lactancia en adelante y por último, las vacas en proceso de secado o con mastitis subclínica. La rutina de ordeño consistió en lavado de los pezones de la ubre, despunte y prueba de paño negro, sellado de las tetas y desinfección de las pezoneras en una solución de yodo al 3\% entre vaca y vaca; además, mensualmente se realizó la prueba de California para detectar mastitis subclínica. El secado de las vacas se efectuó en forma intermitente y se suspendió el suministro de alimento balanceado. Se detectaron estros una hora por la mañana y otra por la tarde. Las vacas con más de 60 días posparto y que no hubieran manifestado celo se palparon por vía rectal para determinar la causa y aplicar una medida correctiva. Se registró el peso mensualmente, su producción de leche diaria y eventos reproductivos de todas las vacas en producción. Las de descarte fueron vendidas para su sacrificio en rastro con un peso y precio promedio de $400 \mathrm{~kg}$ y $\$ 12.40$.

\section{Análisis estadístico}

Se realizó un ANDEVA mediante el PROC GLM del programa estadístico SAS (Littell et al. 2002). En un modelo completamente al azar, se evaluaron los efectos de genotipo $(G)$, en donde se tomaron los datos productivos de diferentes proporciones de raza europea y cebú: 80 a 99\% Holstein (Hs), 60 a 79\% Hs, 80 a 99\% Suizo Pardo (SP) y 60 a $79 \%$ SP, sexo y época, y sus interacciones sobre peso al nacimiento (PNAC), ganancia diaria de peso al destete (GDP1) y el peso al destete en machos y hembras. En las vaquillas se evaluó el efecto raza (R) y edad a la concepción y al primer parto. En las vacas se evaluó el efecto de R, época de parto (EP), y sus interacciones sobre la producción por lactancia (PL), días en lactancia (DLAC), días del parto a la concepción, período interparto (PINT) y producción día interparto (PDI), con el G como efecto fijo y época del año como covariable y sus interacciones sobre la variable dependiente PL. 
Todas éstas se presentan junto con su Error Estándar de la Media (EEM).

Para la evaluación económica se utilizó el análisis de ingresos, en el que se emplearon precios corrientes de mercado, la relación beneficio costo y tasa de retorno al capital (TIR). El cálculo del costo del litro de leche y kilogramo de carne se realizó por el método de prorrateo, distribuyendo los costos totales anuales del sistema, de acuerdo a la proporción porcentual con que incide el valor de la venta de cada uno de estos productos en el valor total anual de los ingresos, dividiendo los resultados entre los litros de leche y kilogramos de carne producidos (Schaefer 1981).

Se analizaron los registros de los inventarios (activos) así como los ingresos y egresos de los años 2002 y 2003, para determinar la inversión inicial, costos de producción de leche y carne, ingresos, utilidad, rentabilidad y punto de equilibrio (Aguilar y Rueda 1999).

\section{RESULTADOS Y DISCUSIÓN}

\section{Evaluación productiva}

Para los machos el efecto de genotipo fue significativo $(\mathrm{P}<0,05)$, para PNAC las diferencias fueron a favor de los animales 80 a $99 \%$ SP y 60 a $79 \%$ Hs con $41,62 \mathrm{~kg}$; EEM 2,852 y 40,63 kg(EEM 2,900, respectivamente; y para peso al destete las diferencias fueron a favor de los animales SP 60 a 79\% y Hs con 119,54 kg
EEM 11,257 y 107,36 kg; EEM 5,782 respectivamente (Cuadro 1), mientras que la GDP presentó diferencias significativas para el GSP $(\mathrm{P}<0,05)$.

Al comparar la interacción genotipo por sexo de machos y hembras se observaron diferencias $(\mathrm{P}<0,05)$ para los machos con 40,61 kg (EEM 2,716) y para las hembras con 30,76 $\mathrm{kg}$ (EEM 3,823) al nacimiento. Los pesos al nacimiento de hembras y machos coinciden con trabajos reportados desde finales de los 70 hasta la fecha en el trópico húmedo (Ochoa 1979, Lemos et al. 1984, Cunningham y Syrstad 1987, Castañeda et al. 2006), y sólo para GDP y peso al destete (PDES) para machos SP, los resultados obtenidos en el presente trabajo fueron superiores a los informados por Ochoa (1979), que fueron de $0,944 \mathrm{~kg}$ y $119,74 \mathrm{~kg}$ vs 0,441 $\mathrm{kg}$ y 77,20 kg, respectivamente. Los pesos promedio al nacimiento de $33,41 \mathrm{~kg}$ en hembras de los genotipos Hs y SP, fueron menores a los reportados por Correa (1986) quien indica 38,4 kg de PNAC; sin embargo, para PDES el promedio obtenido en este trabajo fue ligeramente superior al indicado por el mismo autor, $89,31 \mathrm{~kg}$ contra $85 \mathrm{~kg}$, respectivamente. Por otro lado, la GDP del destete a los 18 meses del genotipo Hs $(0,351 \mathrm{~kg})$ fue inferior en becerras de la misma raza, en pastoreo de gramíneas en clima templado, que obtuvieron GDP de 0,850 kg (Reyes et al. 1999); el efecto del clima y la baja calidad nutritiva de los forrajes tropicales pudo ser la causante de esta baja ganancia.

Los mejores pesos al nacimiento por genotipo y sexo $(\mathrm{P}<0,05)$ fueron para los machos 80 a $99 \%$ SP con 40,61 kg (EEM 2,716), seguidos por los machos 60 a

Cuadro 1. Pesos y ganancias de peso diarias de becerros del nacimiento al destete por genotipo. Módulo de Lechería Tropical del C.E. La Posta. INIFAP. México. 2002-2003.

\begin{tabular}{lcccccc}
\hline Genotipo & $\begin{array}{c}\text { Nacimiento } \\
(\mathbf{k g})\end{array}$ & $\begin{array}{c}\text { Error estándar } \\
\text { de la media }\end{array}$ & $\begin{array}{c}\text { Ganancia diaria } \\
\text { de peso }(\mathbf{k g})\end{array}$ & $\begin{array}{c}\text { Error estándar } \\
\text { de la media }\end{array}$ & $\begin{array}{c}\text { Destete (kg) } \\
\text { Error estándar } \\
\text { de la media }\end{array}$ \\
\hline Holstein $(\mathrm{Hs})$ & $30,45 \mathrm{a}$ & 2,69 & $0,598 \mathrm{a}$ & 0,075 & $84,30 \mathrm{ab}$ & 5,36 \\
$80 \mathrm{a} 99 \% \mathrm{Hs}$ & $34,68 \mathrm{ab}$ & 1,76 & $0,557 \mathrm{a}$ & 0,05 & $84,77 \mathrm{~b}$ & 3,52 \\
60 a 79\% Hs & $40,63 \mathrm{~b}$ & 2,90 & $0,742 \mathrm{ab}$ & 0,08 & $107,36 \mathrm{ab}$ & 5,78 \\
Suizo Pardo (SP) & $34,56 \mathrm{ab}$ & 5,64 & $0,944 \mathrm{~b}$ & 0,16 & $119,54 \mathrm{a}$ & 11,25 \\
80 a 99\% SP & $41,62 \mathrm{~b}$ & 2,85 & $0,714 \mathrm{a}$ & 0,08 & $105,82 \mathrm{ab}$ & 5,69 \\
60 a 79\% SP & $28,55 \mathrm{ab}$ & 5,59 & $0,663 \mathrm{a}$ & 0,16 & $88,25 \mathrm{ab}$ & 11,15 \\
\hline
\end{tabular}

ab Columnas con diferentes literales son estadísticamente diferentes, según prueba de Tukey $(\mathrm{P}<0,05)$. 
Cuadro 2. Pesos, ganancia diaria de peso y edad a la concepción de novillonas ( $\approx 280 \mathrm{~kg}$ ) por genotipo. Módulo de Lechería Tropical del Campo Experimental La Posta. INIFAP. México. 2002-2003.

\begin{tabular}{|c|c|c|c|c|c|c|c|c|}
\hline Genotipo & $\begin{array}{l}\text { Peso al naci- } \\
\text { miento (kg) }\end{array}$ & $\begin{array}{l}\text { Ganancia dia- } \\
\text { ria de peso del } \\
\text { nacimiento al } \\
\text { destete }(\mathrm{kg})\end{array}$ & $\begin{array}{c}\text { Peso al } \\
\text { destete } \\
\text { (kg) }\end{array}$ & $\begin{array}{l}\text { Ganancia dia- } \\
\text { ria de peso del } \\
\text { destete al año } \\
(\mathrm{kg})\end{array}$ & $\begin{array}{c}\text { Peso al } \\
\text { año }(\mathbf{k g})\end{array}$ & $\begin{array}{l}\text { Ganancia dia- } \\
\text { ria de peso del } \\
\text { año a los } 18 \\
\text { meses }(\mathrm{kg})\end{array}$ & $\begin{array}{c}\text { Peso a los } \\
18 \text { meses } \\
(\mathrm{kg})\end{array}$ & $\begin{array}{c}\text { Edad a la } \\
\text { concepción } \\
\text { (meses) }\end{array}$ \\
\hline Holstein & $\begin{array}{l}34,32 \mathrm{a} \\
*(2,71)\end{array}$ & $\begin{array}{c}0,643 \text { a } \\
(0,06)\end{array}$ & $\begin{array}{c}92,28 \mathrm{a} \\
(4,91)\end{array}$ & $\begin{array}{c}0,240 \mathrm{a} \\
(0,07)\end{array}$ & $\begin{array}{c}159,53 \mathrm{a} \\
(11,86)\end{array}$ & $\begin{array}{c}0,351 \mathrm{ab} \\
(0,06)\end{array}$ & $\begin{array}{c}210,47 \mathrm{ab} \\
(16,57)\end{array}$ & $\begin{array}{c}34,67 \mathrm{a} \\
(3,00)\end{array}$ \\
\hline $80-99 \% \mathrm{Hs}$ & $\begin{array}{c}30,04 \mathrm{a} \\
(2,23)\end{array}$ & $\begin{array}{c}0,620 \mathrm{a} \\
(0,05)\end{array}$ & $\begin{array}{c}85,56 \mathrm{a} \\
(3,77) \\
\end{array}$ & $\begin{array}{c}0,363 \mathrm{a} \\
(0,05)\end{array}$ & $\begin{array}{c}183,85 \mathrm{ab} \\
(9,11)\end{array}$ & $\begin{array}{c}0,376 \mathrm{ab} \\
(0,05)\end{array}$ & $\begin{array}{c}257,43 \text { bc } \\
(12,16)\end{array}$ & $\begin{array}{c}33,38 \mathrm{a} \\
(2,30) \\
\end{array}$ \\
\hline $60-79 \% \mathrm{Hs}$ & $\begin{array}{c}31,32 \mathrm{a} \\
(6,53)\end{array}$ & $\begin{array}{c}0,970 \mathrm{a} \\
(0,15)\end{array}$ & $\begin{array}{c}120,28 \text { a } \\
(11,92)\end{array}$ & $\begin{array}{c}0,110 \mathrm{a} \\
(0,16)\end{array}$ & $\begin{array}{c}150,15 \text { a } \\
(27,79)\end{array}$ & $\begin{array}{c}0,15 \mathrm{ab} \\
(0,15)\end{array}$ & $\begin{array}{c}146,81 \mathrm{ac} \\
(37,34)\end{array}$ & $\begin{array}{c}33,92 \text { a } \\
(7,03)\end{array}$ \\
\hline $50 \% \mathrm{Hs}$ & $\begin{array}{c}40,32 \mathrm{a} \\
(8,44)\end{array}$ & $\begin{array}{c}0,644 \mathrm{a} \\
(0,20) \\
\end{array}$ & $\begin{array}{l}98,28 \mathrm{a} \\
(14,85)\end{array}$ & $\begin{array}{c}0,604 \mathrm{a} \\
(0,21) \\
\end{array}$ & $\begin{array}{c}260,53 \mathrm{~b} \\
(35,90)\end{array}$ & $\begin{array}{c}-0,055 \mathrm{ab} \\
(0,19)\end{array}$ & $\begin{array}{c}241,47 \mathrm{ab} \\
(46,27)\end{array}$ & $\begin{array}{c}26,67 \mathrm{a} \\
(9,08) \\
\end{array}$ \\
\hline $\begin{array}{l}\text { Suizo Pardo } \\
\text { SP }\end{array}$ & $\begin{array}{c}32,50 \mathrm{a} \\
(3,92)\end{array}$ & $\begin{array}{c}0,600 \mathrm{a} \\
(0,09) \\
\end{array}$ & $\begin{array}{c}86,33 \text { a } \\
(6,88) \\
\end{array}$ & $\begin{array}{c}0,287 \mathrm{a} \\
(0,10) \\
\end{array}$ & $\begin{array}{c}163,59 \mathrm{a} \\
(16,62)\end{array}$ & $\begin{array}{c}0,240 \mathrm{~b} \\
(0,09) \\
\end{array}$ & $\begin{array}{c}205,89 \mathrm{a} \\
(21,45) \\
\end{array}$ & $\begin{array}{c}25,73 \mathrm{a} \\
(4,20) \\
\end{array}$ \\
\hline $80-99 \%$ SP & $\begin{array}{c}33,10 \mathrm{a} \\
(4,54)\end{array}$ & $\begin{array}{c}0,675 \mathrm{a} \\
(0,16) \\
\end{array}$ & $\begin{array}{l}92,80 \mathrm{a} \\
(12,08) \\
\end{array}$ & $\begin{array}{c}0,320 \mathrm{a} \\
(0,17) \\
\end{array}$ & $\begin{array}{c}178,94 \mathrm{ab} \\
(29,21)\end{array}$ & $\begin{array}{c}0,487 \mathrm{ab} \\
(0,16)\end{array}$ & $\begin{array}{c}281,78 \mathrm{ab} \\
(38,12)\end{array}$ & $\begin{array}{c}37,02 \mathrm{a} \\
(7,39) \\
\end{array}$ \\
\hline $60-79 \%$ SP & $\begin{array}{c}34,04 \mathrm{a} \\
(5,70)\end{array}$ & $\begin{array}{c}0,427 \text { a } \\
(0,13)\end{array}$ & $\begin{array}{c}72,23 \mathrm{a} \\
(9,97)\end{array}$ & $\begin{array}{c}0,469 \text { a } \\
(0,14)\end{array}$ & $\begin{array}{c}195,18 \mathrm{ab} \\
(24,10)\end{array}$ & $\begin{array}{c}0,412 \mathrm{ab} \\
(0,13)\end{array}$ & $\begin{array}{c}265,93 \mathrm{ab} \\
(32,882)\end{array}$ & $\begin{array}{c}29,21 \text { a } \\
(6,09)\end{array}$ \\
\hline $50 \% \mathrm{SP}$ & $\begin{array}{c}35,86 \text { a } \\
(6,89)\end{array}$ & $\begin{array}{c}0,630 \mathrm{a} \\
(0,16)\end{array}$ & $\begin{array}{l}93,09 \mathrm{a} \\
(11,81)\end{array}$ & $\begin{array}{c}0,399 \mathrm{a} \\
(0,16)\end{array}$ & $\begin{array}{c}201,37 \mathrm{ab} \\
(28,55)\end{array}$ & $\begin{array}{c}0,707 \text { a } \\
(0,15)\end{array}$ & $\begin{array}{c}324,44 \mathrm{ab} \\
(36,96)\end{array}$ & $\begin{array}{c}27,99 \mathrm{a} \\
(7,22)\end{array}$ \\
\hline
\end{tabular}

ab Columnas con diferentes literales son estadísticamente diferentes, según prueba de Tukey $(\mathrm{P}<0,05)$.

* Valores dentro del paréntesis corresponden al error estándar de la media, para cada variable.

$79 \%$ Hs con 39,71 kg (EEM 2,748), las hembras SP con $36,94 \mathrm{~kg}$ (EEM 2,971) y los machos 80 a $99 \% \mathrm{Hs}$ con 35,40 kg (EEM 1,735); en lo que se refiere a GDP, se observaron las menores ganancias en las hembras SP con $0,493 \mathrm{~kg}($ EEM 0,083) y en los machos 80 a $99 \% \mathrm{Hs}$ con $0,580 \mathrm{~kg}($ EEM 0,049). En cuanto a pesos al destete, los resultados mostraron un mayor peso $(\mathrm{P}<0,05)$ para los machos SP, 60 a $79 \%$ Hs y 80 a $99 \%$ SP que para las hembras de los mismos genotipos con 118,74 $\mathrm{kg}$ (EEM 10,180), 104,04 kg (EEM 4,972), 102,59 kg (EEM 4,909), 74,63 kg (EEM 5,378), 95,06 kg (EEM 3,741) y $87,42 \mathrm{~kg}($ EEM 5,677$)$ respectivamente $(\mathrm{P}<0,05)$; sin embargo, los genotipos Hs y aquellos con 80 a $99 \%$ de esta sangre no mostraron diferencias estadísticas entre sexos para peso al destete $(\mathrm{P}>0,05)$.

El efecto de genotipo para pesos y GDP del nacimiento al destete, de éste al año, del año a los 18 meses y la edad a la concepción de novillonas (Cuadro 2), no mostraron diferencias estadísticas para la GDP1 ( $\mathrm{P}>0,05)$; el genotipo 60 a $79 \%$ Hs presentó el mayor peso al destete con 120,28 kg (EEM 11,918), seguido del $50 \%$ Hs con 98,28 kg (EEM 14,855), mientras que los genotipos restantes obtuvieron pesos por debajo de los 95 $\mathrm{kg}(\mathrm{P}<0,05)$. En la GDP2 no se presentaron diferencias significativas $(\mathrm{P}>0,05)$, mientras que para peso al año, GDP3 y peso a 18 meses se observaron diferencias entre genotipos; los mayores obtenidos al año fueron para los genotipos 50\% SP y $50 \%$ Hs con 260,53 kg (EEM $35,907)$ y $201,37 \mathrm{~kg}($ EEM 28,553$)$ respectivamente, las mejores ganancias estuvieron a favor de la raza $50 \% \mathrm{SP}$ con $0,707 \mathrm{~kg}$ (EEM 0.154), en tanto que los genotipos restantes tuvieron una ganancia por debajo de los 0,500 $\mathrm{kg}(\mathrm{P}<0,05)$; a los 18 meses, el mejor peso se registró en el genotipo 50\% SP con 324,44 kg (EEM 36,966). 
Para la edad a la concepción (ECONC) no se observaron diferencias significativas $(\mathrm{P}>0,05)$, siendo la edad promedio de 2,6 años. Se ha determinado que las hembras provenientes de las cruzas entre las razas cebuinas y europeas, en condiciones de trópico húmedo, inician la pubertad a edad más temprana (457 a 477 días) que las razas puras (583 días) (Calderón et al. 1993). No obstante, las novillonas SP presentan la pubertad a menor peso $(273 \mathrm{~kg})$ que las Hs x C (305 kg), SP x C (303 kg) y Simmental x C (301 kg), bajo condiciones de manejo de doble propósito. Con base en lo anterior, es posible que las condiciones de semiestabulación y pastoreo nocturno implementadas con los animales de este estudio, permitieran que los pura sangre SP tuvieran una edad a la concepción similar a los cruzados, ya que sólo se habían evaluado animales puros con cruzados en condiciones de manejo de pastoreo extensivo.

En Yucatán, el promedio de edad a la concepción obtenido con novillas alimentadas con forraje de corte Taiwán (Pennisetum purpureum, var. Taiwan) al 2\% de materia seca en relación a su peso vivo, y suplementadas con un alimento balanceado de $16 \%$ de PC, fue menor que el obtenido en este trabajo $(19,33 \mathrm{vs}$ 31,37 meses, respectivamente) (Hau y Santos 1999). Es importante señalar que los objetivos de estos autores eran solamente lograr con una excelente alimentación el desarrollo de novillas sin importar los costos, mientras que en este trabajo se evalúa el desarrollo de todo el hato con el factor económico de rentabilidad sobre costos totales y el objetivo de tener novillonas de por lo menos 2,5 años de edad al parto; este último se alcanzó en los genotipos SP y 50\% Hs (ECONC promedio de 2,2 años), a diferencia del resto de los genotipos, en los que la edad al parto es de 3,5 años (ECONC promedio de 2,7 años para todas la hembras de este estudio).

El efecto de genotipo para la PL fue significativo y las vacas con mayor producción fueron las 80 a $99 \% \mathrm{Hs}(\mathrm{P}<0,05)$ y las menos productivas las $50 \%$ Hs, con 3796,10 litros (EEM 246,352) vs 836 litros (EEM 779,033), respectivamente; a su vez, los DLAC no mostraron diferencias estadísticas $(\mathrm{P}>0,05)$. En la producción/día/lactancia (PDL) los genotipos sobresalientes fueron el 80 a 99\% Hs con 11,44 litros y el Hs con 11,12 litros, (Cuadro 3); la producción/día/interparto (PDI) no mostró diferencia estadística $(\mathrm{P}>0,05)$, sin embargo el genotipo Hs fue el que mostró la mayor producción con 8,57 litros (EEM 1,354) y el $50 \%$ Hs la menor, con 2,70 litros (EEM 1,914). El periodo interparto más elevado fue para el grupo 60 a 79\% Hs con 502 días (EEM 67,114), seguido del grupo 80 a 99\% Hs con 428,60 días (EEM 51,986), mientras que el más bajo fue para el genotipo $50 \%$ Hs con 305 días (EEM 116,245). De acuerdo a estos resultados, los parámetros PL, DLACT y PDL de la raza SP $(2934,25$ litros, 317,75 días y 9,33 litros, respectivamente), fueron similares a los reportados por Bonilla y Bustamante (1997) en clima tropical subhúmedo con 2991 litros, 302 días y 9,90 litros. Por su parte, para la raza

Cuadro 3. Parámetros por genotipo de vacas en producción. Módulo de Lechería Tropical del Campo Experimental La Posta. INIFAP. México. 2002-2003.

\begin{tabular}{|c|c|c|c|c|c|c|c|c|c|c|}
\hline Genotipo & $\begin{array}{c}\text { Días en } \\
\text { lactancia }\end{array}$ & *EEM & $\begin{array}{c}\text { Producción } \\
\text { por } \\
\text { lactancia }\end{array}$ & EEM & $\begin{array}{l}\text { Produc- } \\
\text { ción/día/ } \\
\text { lactancia }\end{array}$ & EEM & $\begin{array}{c}\text { Produc- } \\
\text { ción/día/ } \\
\text { interparto }\end{array}$ & EEM & $\begin{array}{c}\text { Periodo } \\
\text { interparto }\end{array}$ & EEM \\
\hline Holstein (Hs) & $310,00 \mathrm{a}$ & 27,28 & $3425,40 a b$ & 348,39 & $11,12 \mathrm{ab}$ & 0,69 & $8,57 \mathrm{a}$ & 1,35 & $417,50 \mathrm{a}$ & 82,2 \\
\hline$(80-99 \%) \mathrm{Hs}$ & $331,50 \mathrm{a}$ & 19,29 & $3796,10 \mathrm{~b}$ & 246,35 & $11,44 \mathrm{~b}$ & 0,49 & $8,36 \mathrm{a}$ & 0,85 & $428,60 \mathrm{a}$ & 52,0 \\
\hline$(60-79 \%) \mathrm{Hs}$ & $292,25 \mathrm{a}$ & 30,50 & $2911,00 \mathrm{ab}$ & 389,51 & $9,83 \mathrm{ab}$ & 0,77 & $7,07 \mathrm{a}$ & 1,10 & $502,00 \mathrm{a}$ & 67,1 \\
\hline$(50 \%) \mathrm{Hs}$ & $133,00 \mathrm{a}$ & 61,01 & 836,00 a & 779,03 & $6,30 \mathrm{ab}$ & 1,55 & $2,70 \mathrm{a}$ & 1,91 & $305,00 \mathrm{a}$ & 116 \\
\hline Suizo Pardo (Sp) & $317,75 \mathrm{a}$ & 30,50 & $2934,25 \mathrm{ab}$ & 389,51 & $9,33 \mathrm{ab}$ & 0,78 & $7,16 \mathrm{a}$ & 1,10 & $428,33 \mathrm{a}$ & 67,1 \\
\hline$(60-79 \%) \mathrm{Sp}$ & $177,50 \mathrm{a}$ & 43,14 & $1358,50 \mathrm{a}$ & 550,86 & $7,25 \mathrm{a}$ & 1,10 & $5,40 \mathrm{a}$ & 1,91 & $383,00 \mathrm{a}$ & 116 \\
\hline
\end{tabular}

ab Columnas con diferentes literales son estadísticamente diferentes, según prueba de Tukey $(\mathrm{P}<0,05)$.

* EEM: Error estándar de la media. 
Hs los parámetros fueron mejores a los reportados por los mismos autores (3425,40 vs 3295 litros, 310 vs 367 días y 11,12 vs 8,97 litros, respectivamente).

En Arizona, se ha trabajado con vacas Holstein puras bajo condiciones de estrés calórico con tres tratamientos: 1) Sólo sombra, 2) Enfriamiento basado en aspersores y abanicos + sombra y 3 ) Enfriamiento evaporativo Korral Kool + sombra, obteniendo una producción diaria de leche de 31,0, 39,1 y 37,9 litros respectivamente, la cual fue muy superior a la de 11,12 litros que se obtuvo en este trabajo para la raza pura sangre Hs (Correa et al. 1999); por otra parte, los resultados que se obtuvieron de los parámetros producción diaria de leche y días en lactancia en las diferentes cruzas (Europeo por Cebú) fueron superiores a los presentados por Osorio y Segura (1999) en Yucatán, donde la PDL y los DLACT fueron de 5,9 litros y 213 días, respectivamente.

El efecto de época de mayor predominancia conocidos como nortes, secas y lluvias en la costa del Golfo de México, y que correspondería a invierno, primavera y verano para número de vacas en lactancia y producción láctea por semana/época (Cuadro 4), mostró que para número de vacas/semana existió diferencia significativa a favor de la época de secas $(\mathrm{P}<0,01)$ con 23,74 vacas (EEM 0,294), seguida de la época de nortes con 21,98 vacas (EEM 0,291 ) y época de lluvias con 21,15 vacas (EEM 0,291). La producción/semana también estuvo a favor de la época seca $(\mathrm{P}<0,01)$ con 1670,67 litros (EEM 27,368), seguida de

Cuadro 4. Número de vacas en lactancia y producción láctea por semana/época. Módulo de Lechería Tropical del Campo Experimental La Posta. INIFAP. México. 2002-2003.

\begin{tabular}{lcc}
\hline Época & No. vacas/semana & Producción/semana \\
\hline Nortes & $21,98 \mathrm{a}$ & $1598,94 \mathrm{ab}$ \\
& $*(0,291)$ & $(27,180)$ \\
\hline Secas & $23,74 \mathrm{~b}$ & $1670,67 \mathrm{a}$ \\
& $(0,294)$ & $(27,368)$ \\
\hline \multirow{2}{*}{ Lluvias } & $21,15 \mathrm{a}$ & $1552,84 \mathrm{~b}$ \\
& $(0,291)$ & $(27,173)$ \\
\hline
\end{tabular}

Columnas con diferentes literales son estadísticamente diferentes, según prueba de Tukey $(\mathrm{P}<0,01)$.

* Valores dentro del paréntesis corresponden al error estándar de la media, para cada variable. la época de nortes con 1598,94 litros (EEM 27,180) y lluvias con 1552,84 litros (EEM 27,173). Al obtener el promedio de vaca línea/semana se observó que el efecto de época del año afectó sólo al número de vacas en producción, pero no de leche, ya que las tres diferentes épocas tienen un promedio de vaca línea de $10,39,10,05$ y 10,49 litros respectivamente $(\mathrm{P}>0,05)$. Lo anterior se debió a que durante la época de lluvias se gestó el mayor número de animales $(60 \%)$ y éstos parieron en la época de secas, aumentando el número de animales en producción, lo que coincide con lo mencionado por Aluja et al. (1998), que reportan que el $41 \%$ de los partos ocurren en el periodo de sequía (marzo-abril), lo cual indica que las vacas concibieron principalmente en la época de lluvias (junio-octubre).

Con relación a la meta productiva de la carga animal por hectárea, se logró alcanzar este parámetro con $5 \mathrm{UA} / \mathrm{ha}$, con una producción anual de 82769 litros de leche en 20 ha de pastoreo, lo que correspondió a 4138 litros/ha. Esto se pudo lograr básicamente por la suplementación con concentrado y forraje de corte durante la estabulación del ganado y de forraje de corte durante la época de sequía.

\section{Evaluación económica}

La inversión (a precios del año 2003) fue de $\$ 884427$ ó US\$78 $660^{4}$, de la cual el ganado representó $36,06 \%$, seguido del terreno con $16,91 \%$ y $47,03 \%$ para los otros factores.

\section{Producción de leche y carne}

En el periodo de estudio se produjeron 82769 litros de leche, y la producción total de carne fue de $16592 \mathrm{~kg}$ la cual sirve para determinar los ingresos reales tal y como se presentaron a la hora de su venta; es necesario que en éste se haga una separación en lo que fue el ingreso real por venta de los productos (Simpson 1989).

\section{Ingreso}

En este sistema de producción, los ingresos fueron por la venta de leche y carne. En 2003, el ingreso por

4 Tipo de cambio de 11,2436 pesos mexicanos por un dólar americano, durante el año 2003. 
la venta de ambos productos fue de US\$28427,7 del cual, $82 \%$ correspondió a la venta de leche y $18 \%$ a la de carne (Cuadro 5).

Cuadro 5. Ingresos por concepto de leche y carne. Módulo de Lechería Tropical del C.E. La Posta. INIFAP. 2002-2003.

\begin{tabular}{lc}
\hline Concepto & US\$ \\
\hline Leche & 23409 \\
Carne & 5018,5 \\
Total & $\mathbf{2 8 4 2 7 , 7}$ \\
\hline
\end{tabular}

Costos de producción total de productos leche $\mathbf{y}$ carne de bovino

Dentro de los costos de producción el concepto de mayor gasto fue el alimento concentrado, con $24,35 \%$ seguido de la mano de obra con $15,72 \%$.

\section{Costos de producción por prorrateo}

Se determinó el costo por litro de leche y kilogramo de carne, del capital estos costos fueron de US $\$ 0,29$ y US $\$ 1,19$, respectivamente (Cuadro 6).

\section{Margen de ganancia}

Para calcular el margen de ganancia se procedió a hacer una operación de la relación beneficio costo obteniéndose para leche 1:1.12 y para carne $1: 1.1$. Si observamos a la explotación en forma integral, su relación beneficio costo sería de 1:1.11 el cual es mayor en casi dos veces al interés que ofrecen los bancos.

\section{Utilidad bruta y neta}

La utilidad bruta de la explotación fue de US\$28 427,7 y sus costos totales de US\$25 456,2, obteniéndose una utilidad neta de US\$2971,6.

\section{Rentabilidad}

La rentabilidad sobre el costo total (CT) fue de $11 \%$, con respecto a los productos generados en la explotación (leche y carne).
Cuadro 6. Costo por unidad de producto (por prorrateo). Módulo de Lechería Tropical del Campo Experimental La Posta. INIFAP. 2002-2003.

\begin{tabular}{lcc}
\hline \multicolumn{1}{c}{ Concepto } & $\begin{array}{c}\text { Leche } \\
\mathbf{( 8 2 \% )}\end{array}$ & $\begin{array}{c}\text { Carne } \\
\mathbf{( 1 8 \% )}\end{array}$ \\
\hline riable (US\$) & 4224,8 & 927,4 \\
Alimento concentrado & 6544,0 & 1436,7 \\
Leche para becerros & 2327,2 & 510,9 \\
Fertilizantes y herbicidas & 43,9 & 9,6 \\
Servicio veterinario & 2625,5 & 576,3 \\
Desparasitantes & 161,2 & 35,4 \\
Vacunas & 88,7 & 19,5 \\
Otras medicinas & 269,8 & 59,2 \\
Inseminación artificial & 65,1 & 14,3 \\
Combustibles & 2719,9 & 597,1 \\
Reparaciones & 412,4 & 90,5 \\
Útiles y equipo & 412,2 & 90,5 \\
Otros & 979,1 & 214,5 \\
\hline Total (US\$) & $\mathbf{2 0 ~ 8 7 3 , 9}$ & $\mathbf{4 5 8 2 , 3}$ \\
\hline
\end{tabular}

\section{Punto de equilibrio en leche y carne}

El punto de equilibrio (PE) para leche y carne se alcanzó, con una diferencia a favor del 12 y $324 \%$ respectivamente, tomando como referencia al punto de equilibrio. El gran porcentaje de diferencia entre el punto de equilibrio en carne y la diferencia producida se debió a que en el primer año se vendieron muchas vacas de descarte (Cuadro 7).

Finalmente, podemos concluir que las cruzas Hs/ $\mathrm{C}$ y SP/C muestran un mejor desarrollo que las razas pura sangre Hs y SP. Los mejores pesos al destete de los machos fueron para los SP y sus cruzas con C. El parámetro de ECONC en las novillonas SP es mejor que sus cruzas con Cebú mientras que las novillonas $50 \%$ Hs superaron a las Hs de pura sangre.

Mediante manejo de lechería tropical el mejor comportamiento productivo fue para las vacas $80-99 \%$ $\mathrm{Hs}$, y el menor para las $50 \% \mathrm{Hs}$. Al igual que los sistemas de doble propósito, en lechería tropical especializada las vacas conciben principalmente en la época de lluvias, durante la cual el ambiente y la disponibilidad de forraje son favorables para la presentación de los estros. 
Cuadro 7. Punto de equilibrio (cantidad producida sin perdidas o ganancias) en la producción de leche y carne. Módulo de Lechería Tropical del C. E. La Posta. INIFAP. 2002-2003.

\begin{tabular}{lrr}
\hline Concepto & Leche & \multicolumn{1}{c}{ Carne } \\
\hline Costos totales (\$) & 20873,80 & 4582,30 \\
Precio de venta (\$) & 0,28 & 1,17 \\
Producción anual (L y kg) & 7361,4 & 16592 \\
Punto de equilibrio (L y kg) & 73804 & 3909 \\
Diferencia (L y kg) & 8965 & 12683 \\
\hline
\end{tabular}

Con un mejor manejo de potreros y el pastoreo rotacional nocturno, se logró incrementar la producción láctea y la carga animal/hectárea/año a más del cien por ciento del promedio de la región.

\section{LITERATURA CITADA}

Aguilar, BU; Rueda, MBL. 1999. Metodología para la evaluación económica en ranchos ganaderos de Doble Propósito. In Manual del manejo de ganado bovino de doble propósito en el trópico. Campo Experimental La Posta. CIRGOC. INIFAP. SAGAR. Veracruz, México. p. $124-140$.

Aluja, A; Acosta, R; Pulido, A. 1998. Comportamiento del ganado Holstein x Cebú en sistemas de lechería tropical. In Memorias IV foro de análisis de los recursos genéticos: Ganadería bovina de doble propósito. Tabasco, México. p. 85-89.

Barradas, LHV; Román, PH; Monroy, AV. 1979. Comportamiento de becerros de razas lecheras en diferentes sistemas de alojamiento en clima tropical. Técnica Pecuaria en México 37:29-33

Bonilla, CJA; Bustamante, GJJ. 1997. Producción de leche con vacas Suizo Pardo y Holstein en clima tropical. In: Memoria XXXIII Reunión Nacional de Investigación Pecuaria. Veracruz, México. 169 p.

Calderón, RR; Villa-Godoy, A; Zapata, SLE; Lagunes, LJ. 1993. Actividad ovárica en becerras Suizo Pardo y Cebú en clima tropical Af (c). I. Inicio de la pubertad y su correlación con la primera ovulación. In Memorias Reunión Nacional de Investigación Pecuaria Jalisco 1993. INIFAP. Jalisco, México. 160 p.

Castañeda, MOG; Ávila, DA; Vinay, VJC. 2006. Comportamiento productivo de ganado cruzado europeo por cebú en clima tropical. In Memorias XVI Reunión Internacional Sobre Producción de Carne y Leche en Clima Cálido. Baja California, México. p. $195-$ 199.

Castro, LCJ; Sánchez, RG; Iruegas, ELF; Saucedo, LG. 2001. Tendencias y oportunidades de desarrollo de la red leche en México. México, DF. FIRA Boletín Informativo. Volumen XXXIII. Núm. 317. 9a. época. Año XXX. Septiembre. México, D. F. 65 p.

Correa, CA; Armstrong, DV; Ray, DE; González, GH; Verdugo, ZF. 1999. Respuesta productiva y reproductiva de vacas Holstein bajo estrés térmico a dos diferentes sistemas de enfriamiento. In Memorias XXXV Reunión Nacional de Investigación Pecuaria INIFAP. Yucatán, México. p. 21.

Correa, RJR. 1986. Estudio recapitulativo de la crianza de ganado productor de leche en la etapa de lactancia para el periodo 1970-1983. Tesis Profesional. Universidad Nacional Autónoma de México. México, D.F.

Cunningham, EP; Syrstad, O. 1987. Crossbreeding Bos indicus and Bos taurus for milk production in the tropics. FAO Animal Production Health. Paper No. 68. Rome, Italy. 316 p.

Edmonson, AJ; Lean, IJ; Weaver, LD; Farver, T; Webster, G. 1989. A body condition coring chart for Holstein cows. J. Dairy Sci. 72:68-78.

García, HL; Álvarez, MA; Martínez, BE; Del Valle, RC. 1997. Marco Internacional, Regional e Interrelaciones de los Sistemas Nacionales Lecheros de América del Norte. In Memorias II Seminario Internacional sobre los Sistemas Nacionales Lecheros de América del Norte. México, D.F. p. 2-8.

García, ME. 1989. Apuntes de climatología. 6 ed. Universidad Nacional Autónoma de México (UNAM). México D.F. p. 155.

Hau, CE; Santos, FJ. 1999. Edad y peso a la pubertad en novillas cruzadas alimentadas con pasto Taiwán (Pennisetum purpureum var. Taiwán) y un concentrado. In Memorias XXXV Reunión Nacional de Investigación Pecuaria. Yucatán, México. p. 263.

Lemos, AM; Teodoro, RL; Barbosa, RT; Freitas, AF; Madalena, FE. 1984. Comparative performance of six Holstein Friesian X Guzera Grades in Brazil. Anim. Prod. 38:157-164.

Littell, RC; Stroup, WW; Freund, RJ. 2002. SAS ${ }^{\circledR}$ for Linear Models, 4 ed. Cary, NC: SAS Institute Inc.

Loyola, AJA. 2000. Globalización y desarrollo en México. México, D. F. p. 12-18. 
Nuñez, OJM; Solís, SA; Rodríguez, PCG; Blanco, DR. 1999. Situación actual del productor lechero de menores ingresos en el estado de Jalisco. In XXXV Memorias Reunión Nacional de Investigación Pecuaria. México. p. 333.

Ochoa, OL. 1979. Estudio comparativo de los diferentes métodos de crianza de becerros Pardo Suizo en trópico. Tesis Profesional. México, D.F. Universidad Nacional Autónoma de México. 76 p.

Osorio, AMM; Segura, CJC. 1999. Indicadores de producción de los hatos lecheros del Estado de Yucatán. In Memorias XXXV Reunión Nacional de Investigación Pecuaria. Yucatán, México. p. 318.

Ponce, CP. 2000. Producción y calidad de la leche bajo condiciones del trópico americano. Tropileche 2000. In Memorias III Curso de Capacitación "La Lechería en el Trópico Americano”. FEPALE. CENSA. La Habana, Cuba. p. 1-10.

Reyes, MF; Sánchez, AF; Meráz, DF. 1999. Desarrollo de vaquillas lecheras para reemplazo en praderas en Durango. In Memorias XXXV Reunión Nacional de Investigación Pecuaria. Yucatán, México. p. 94.

Rodríguez, MMA; Castelán, O; Arriaga, JC. 1999. Prácticas de alimentación de vacas lecheras en sistemas de producción de leche en pequeña escala del Valle de Toluca. In Memorias XXXV Reunión Nacional de Investigación Pecuaria. Yucatán, México. p. 264.
Rojas, BB. 1996. Evaluación financiera de un sistema de producción de leche y pie de cría con ganado Suizo Pardo en pastoreo en el subtrópico húmedo. Tesis Profesional. Benemérita Universidad Autónoma de Puebla. Puebla, México. 90 p.

Román, PH; Ortiz, OGA. 1970. Efecto de la cantidad de leche consumida y edad al destete en becerros Holstein bajo condiciones de clima tropical. Técnica Pecuaria en México. p. 24-33.

SAGARPA (Secretaría de Agricultura, Ganadería, Desarrollo Rural, Pesca y Alimentación). 2004. Situación de la producción de leche bovina en México. Boletín informativo. México, D. F. p. 1-19.

SAGAR (Secretaría de Agricultura, Ganadería y Desarrollo Rural). 2000. Centro de Estadística Agropecuaria. Boletín Bimestral de la Leche de Bovino (México). p. 2-21.

Schaefer, KW. 1982. Metodología de análisis de las inversiones en explotaciones agrícolas. Materiales de Capacitación del Instituto de Desarrollo Económico. Banco Mundial. Washington, D.C. USA. p. 342-352.

Simpson, JR. 1989. Optimización: Principios marginales y programación lineal (costos y rendimientos de la raza de doble propósito). In Economía de sistemas de producción ganadera en América Latina. Editorial Agropecuaria Latinoamericana, Inc. Gainesville, Florida, USA. p. 43-46. 